\title{
The Evidence for High-Titer Convalescent Plasma in SARS-CoV-2
}

\author{
David L. Fisher ${ }^{1} \cdot$ Pavel Alin $^{1} \cdot$ Stephen Malnick ${ }^{1}$ (D) \\ Accepted: 15 February 2021 / Published online: 18 February 2021 \\ (C) The Author(s), under exclusive licence to Springer Nature Switzerland AG part of Springer Nature 2021
}

\begin{abstract}
Convalescent plasma therapy has been used successfully in the past to treat respiratory infections. In SARS-CoV-2, there was initially strong evidence in favor of convalescent plasma therapy from a large observational study but the evidence from recent randomized controlled trials has been mixed. However, two of those studies provided convalescent plasma therapy on average 8 days after diagnosis despite earlier data proving that the therapy is most effective when given within 3 days of diagnosis. Another more recent randomized controlled trial found evidence in support of convalescent plasma therapy and we believe that it is no coincidence that they administered convalescent plasma therapy within 3 days of symptom onset. We call for more robustly planned randomized controlled studies to further reliably determine the efficacy of convalescent plasma therapy against SARS$\mathrm{CoV}-2$. Progress has been made with developing a vaccine but there is likely to be a substantial lag in widespread administration of the vaccine, especially in poorer countries. We therefore propose that patients with SARS-CoV-2 infection should be considered for early ambulatory administration of high-dose convalescent plasma in order to reduce the burden of severe SARS-CoV-2 disease.
\end{abstract}

Keywords Convalescent plasma $\cdot$ SARS-CoV-2 $\cdot$ Ambulatory therapy

The SARS-CoV-2 pandemic has affected over 105 million people across the world since the start of the first outbreak in December 2019. Passive antibody transfer using convalescent plasma infusions has been used successfully in the past to treat respiratory infections [1] but in SARS-CoV-2, the evidence has been mixed [2-7].

A large multicenter observational study coordinated by the Mayo Clinic and published in August 2020 comprised 35,322 patients [2]. They found reduced 7-day and 30-day mortality in people administered convalescent plasma therapy. The 7day mortality effect was most pronounced in people administered convalescent plasma therapy within 3 days of SARSCoV-2 diagnosis $(8.7 \%, 8.3-9.2 \%)$ as compared with people who received treatment 4 or more days after diagnosis $(11.9 \%$, 11.4-12.3\%), $P<0.001$. A similar trend was seen with the 30day mortality data. Our experience is consistent with these findings. In our local SARS-CoV-2 unit in a secondary-care healthcare facility, we frequently administer convalescent

This article is part of the Topical Collection on COVID-19

Stephen Malnick

stephen@malnick.net

1 Kaplan Medical Center, Derech Pasternak 1, 76100 Rehovot, Israel plasma therapy and we have often witnessed rapid recoveries when it has been administered promptly following SARSCoV-2 diagnosis [8].

The Mayo Clinic team also recently demonstrated the importance of using high-titer plasma rather than low-titer plasma. This was reflected by a more substantial reduction in mortality within 30 days (relative risk 0.66 , CI 0.48-0.91) [4]. This is consistent with the interim analyses from two trials which administered neutralizing monoclonal antibodies against SARS-CoV-2 within 3 days of diagnosis $[9,10]$. The monoclonal antibody treatments have an antibody titer substantially greater than the titer in convalescent plasma. Interim analysis of both trials showed a reduction in viral load $(-0.53, \mathrm{CI}-0.98$ to 0.08 by day 11$)(-0.56, \mathrm{CI}-1.02$ to 0.10 by day 7) respectively.

Despite the evidence, a Cochrane systematic review was unable to determine if convalescent plasma treatment is effective for SARS-CoV-2 [11]. They identified 19 studies where participants had received convalescent plasma therapy, but the results from only 2 randomized controlled trials were included in their analysis. These 2 studies (both stopped early) comprised only 95 participants who received convalescent plasma therapy. Data from the other studies, which comprised 35,322 participants, were excluded because they were not randomized controlled studies. 
Table 1 Table of studies with positive association between convalescent plasma therapy administration and disease outcome

\begin{tabular}{ll}
\hline Study & Convalescent plasma administration and disease outcome \\
\hline$[2]$ & Mortality rate $8.7 \%[$ CI $8.3-9.2 \%]$ (plasma received within 3 days of diagnosis) vs. 11.9\% \\
& {$[$ CI $11.4-12.3 \%] P<0.001$ (plasma received after 4 days) } \\
{$[4]$} & Lower mortality within 30 days when administered high-titer convalescent plasma vs. \\
& low-titer (relative risk 0.66, CI $0.48-0.91)$ \\
{$[6]$} & Relative risk reduction of developing severe respiratory disease by $48 \%$ (relative risk 0.52 \\
& {$[$ CI $0.29-0.94] P=0.03$ ) } \\
Lower mortality within 60 days when administered high-titer convalescent plasma therapy \\
within 72 h of admission vs. controls (unadjusted hazard ratio $1.93 P=0.02)$
\end{tabular}

This lack of randomized controlled studies has been addressed. However, negative conclusions were drawn from two underpowered studies. One such study was the PLACID trial [3], which was a randomized controlled trial that included people with moderate SARS-CoV-2. They concluded that there was no association between plasma therapy and reduced progression to severe infection or all-cause mortality. During the study, they calculated that to investigate the effectiveness of convalescent plasma therapy, a sample size of 226 participants in each arm of the trial was required. However, only 160 participants received convalescent plasma with "detectable neutralizing antibodies". This fell well short of their target of 226 to achieve a sample size with sufficient power to assess the effectiveness of convalescent plasma. In addition, the median number of days between symptom onset and enrolment to the study was 8 (range 6-11 days). The Mayo Clinic data had shown that the mortality benefit was most pronounced in people administered high-titer convalescent plasma therapy within 3 days of SARS-CoV-2 diagnosis [2, 4]. The Placid trial failed to give sufficient high-titer plasma and was therefore underpowered, and in addition, the administration of the plasma was too delayed to reliably assess its efficacy.

Another randomized controlled trial (Simonovich VA et al. [5]) included 228 participants who received convalescent plasma therapy. They also reported no benefit for convalescent plasma in patients with SARS-CoV-2. Again, the median number of days between symptom onset and study enrolment was 8 , which missed the critical timeframe when plasma therapy is most effective.

The evidence from the Mayo Clinic data in favor of administering convalescent plasma therapy is strong, particularly when it is administered within 3 days of hospitalization for SARS-CoV-2-associated complaints [2]. This has been corroborated recently by another randomized controlled trial which comprised 160 adults (older than 65) with mild or moderate SARS-CoV-2. Eighty people received convalescent plasma and most crucially, it was received within 3 days of the onset of symptoms. They showed a relative risk reduction of developing severe respiratory disease of $48 \%$ (relative risk 0.52 ; confidence interval $0.29-0.94, P=0.03$ ) [6]. Further evidence was found in a prospective study performed in America. The study included 351 people who were transfused high-titer convalescent plasma therapy and they found that the optimal time for administering the therapy was within $44 \mathrm{~h}$ of admission to hospital. There was a mortality benefit after 60 days only among patients who had been transfused with hightiter convalescent plasma therapy within $72 \mathrm{~h}$ of admission (unadjusted hazard ratio $1.93 P=0.02$ ) [7].

In Simonovich V.A. et al. [5], 65.8\% participants were enrolled on the general ward and only $4.8 \%$ were enrolled in the emergency department. Future studies should enroll participants early, preferably in the emergency department so that they receive plasma therapy without delay and within 3 days of diagnosis.

Vaccines for the prevention of SARS-CoV-2 are now becoming available but the pandemic is increasing in prevalence and mutations have been detected. There is likely to be a substantial lag in widespread administration of the vaccine, especially in poorer countries. It is interesting that both of the recent trials with neutralizing monoclonal antibodies administered the therapy to outpatients $[9,10]$. In light of the strong evidence in favor of giving high-titer convalescent plasma therapy within 3 days of diagnosis (Table 1), we propose that patients with SARS-CoV-2 infection should be considered for early ambulatory administration of high-titer convalescent plasma in order to reduce the burden of severe SARS-CoV-2 disease.

Code Availability Not applicable.

Authors' Contribution David Fisher researched the topic and drafted the initial manuscript. Pavel Alin discussed the topic and provided analysis. Stephen Malnick conceived the idea and oversaw the article.

Data Availability Not applicable.

\section{Declarations}

Ethics Approval Not applicable.

Consent to Participate Not applicable. 
Consent for Publication Not applicable.

Competing Interests The authors declare no competing interests.

\section{References}

1. Casadevall A, Scharff MD. Return to the past: the case for antibody-based therapies in infectious diseases. Clin Infect Dis. 1995;21(1):150-61.

2. Joyner MJ, Senefeld JW, Klassen SA, Mills JR, Johnson PW, Theel ES, et al. Effect of convalescent plasma on mortality among hospitalized patients with COVID-19: initial three-month experience. medRxiv. 2020;2020.08.12.20169359. https://doi.org/10. 1101/2020.08.12.20169359.

3. Agarwal A, Mukherjee A, Kumar G, Chatterjee P, Bhatnagar T, Malhotra P, et al. Convalescent plasma in the management of moderate covid-19 in adults in India: open label phase II multicentre randomised controlled trial (PLACID Trial). BMJ. 2020;m3939: 371.

4. Joyner MJ, Carter RE, Senefeld JW, et al. Convalescent plasma antibody levels and the risk of death from Covid-19. NEJM. 2021. https://doi.org/10.1056/NEJMoa2031893.

5. Simonovich VA, Burgos Pratx LD, Scibona $\mathrm{P}$, et al. A randomized trial of convalescent plasma in Covid-19 severe pneumonia. NEJM. 2020. https://doi.org/10.1056/NEHMoa2031304.
6. Libster R, Gonzalo Perez M, Wappner D, et al. Early high-titer plasma therapy to prevent severe Covid-19 in older adults. NEJM. 2021. https://doi.org/10.1056/NEJMoa2033700.

7. Salazar E, Christensen PA, Graviss EA, et al. Significantly decreased mortality in a large cohort of coronavirus disease 2019 (COVID-19) patients transfused early with convalescent plasma containing high-titer anti-severe acute respiratory syndrome coronavirus 2 (SARS-CoV-2) spike protein IgG. Immunopathol Infect Dis. 2021;191:90-107.

8. Fisher DL, Pavel A, Malnick S. Rapid recovery of taste and smell in a patient with SARS-CoV-2 following convalescent plasma therapy. QJM. 2021;hcaa341. https://doi.org/10.1093/qjmed/hcaa341.

9. Chen P, Nirula A, Heller B, et al. SARS-CoV-2 neutralizing antibody LY-CoV555 in outpatients with Covid-19. NEJM. 2021;384: 229-37.

10. Weinreich DM, Sivapalasingam S, Norton T. REGN-COV2, a neutralizing antibody cocktail, in outpatients with Covid-19. NEJM. 2021;384:238-51.

11. Chai KL, Valk SJ, Piechotta V, et al. Convalescent plasma or hyperimmune immunoglobulin for people with COVID-19: a living systematic review. Cochrane Database Syst Rev. 2020;1002(14651858):10.

Publisher's Note Springer Nature remains neutral with regard to jurisdictional claims in published maps and institutional affiliations. 